\title{
Brownian Dynamics
}

National Cancer Institute

\section{Source}

National Cancer Institute. Brownian Dynamics. NCI Thesaurus. Code C70775.

Simulations used to describe the motion of molecules in solution, generally discounting the effects of inertia. 